\title{
A New Neutron Lifetime Experiment with Cold Neutron Beam Decay in Liquid Helium
}

\author{
Wanchun We圈 \\ Kellogg Radiation Laboratory, California Institute of Technology, Pasadena, CA 91125, U.S.A.
}

(Dated: February 6, 2020)

\begin{abstract}
The puzzle remains in the large discrepancy between neutron lifetime measured by the two distinct experimental approaches - counts of beta decays in a neutron beam and storage of ultracold neutrons in a potential trap, namely, the beam method versus the bottle method. In this paper, we propose a new experiment to measure the neutron lifetime in a cold neutron beam with a goal sensitivity of $0.1 \%$ or sub- 1 second. The neutron beta decays will be counted in a liquid helium scintillation detector at $0.5 \mathrm{~K}$, and the neutron flux will be simultaneously monitored by the helium- 3 captures in the same volume. The cold neutron beam must be of wavelength $\lambda>16.5 \AA$ to eliminate scattering with liquid helium.
\end{abstract}

\section{INTRODUCTION}

A precise measurement on the neutron lifetime is crucial to many fundamental questions in particle physics, astrophysics and cosmology, such as CKM unitarity and primordial helium abundance in the Big Bang Nucleosynthesis (BBN). [1, 2] By far, its values obtained from the two distinct methods significantly differ from each other. On one side, the measurement is done in a neutron beam by counting the number of neutrons undergoing beta decays when the neutron flux passes through a defined volume. It is thus called the beam method. The weighted average of the recent two beam lifetime measurements with a proton quasi-Penning trap in the cold neutron $(\mathrm{CN})$ beam is $\tau_{n}=888.0 \pm 2.0 \mathrm{~s}$. [4, 5] On the other side, ultracold neutron (UCN) can be stored in a material box or magneto-gravity trap, and the neutron lifetime is measured by counting the surviving neutrons after a period of storage. It is called the bottle method. The weighted average of several recent bottle lifetime measurements is $\tau_{n}=879.4 \pm 0.6 \mathrm{~s}$. [6] 12] The difference is as large as $8.7 \pm 2.1 \mathrm{~s}(4.1 \sigma)$, most probably due to unaccounted systematic effects in either or both of the methods, yet otherwise it implies new physics. 3. Many further experimental efforts are on the way to address the discrepancy. While existing experiments are upgrading to improve their statistics and searching for hidden systematic effects, new experimental strategies with a dissimilar set of systematic effects are being proposed and carried out. For instance, researchers in J-PARC started a new measurement in the pulsed $\mathrm{CN}$ beam by characterization of the electron recoils in the beta decay events and the helium-3 capture events in a Time Projection Chamber (TPC) filled with gaseous mixture of helium and carbon dioxide. [13] It is a revival of the beam experiment originally proposed by Kossakowski, et al. in 1989. 14] Researchers at Los Alamos National Laboratory are prototyping a beam/bottle hybrid experiment, named UCNProBe, to measure the number of decays and

\footnotetext{
* email: wanchun.wei@caltech.edu
}

helium-3 captures via detection of scintillation in a UCN storage box. 15]

In this paper, we propose a new experimental method with a different combination of existing technologies, in order to resolve the neutron lifetime enigma. The proposed experiment is essentially a beam lifetime measurement. It would count the decay product - electrons, rather than protons, via detection of electron recoil scintillation in superfluid helium at $0.5 \mathrm{~K}$. In order to eliminate neutron scattering with liquid helium, the $\mathrm{CN}$ beam must be of wavelength $\lambda>16.5 \AA$, where kinematics of scattering can never be satisfied. [16] The neutron flux is monitored by helium-3 captures via nuclear recoil scintillation in the same volume of liquid helium. The decay events may be distinguished from the capture events, as the features of scintillation differ between the electron and nuclear recoils. Meanwhile, a precise beta spectrum of neutrons, in addition to the neutron capture peak, will be constructed in a wide energy window of high resolution. A good fit of beta spectrum can separate the overlapping counts of decay events from the capture events, and complement the total counts with the missing number of electrons outside the detectable energy window. In the end, an accurate neutron lifetime can be obtained with a good knowledge of the ratio of capture-to-decay event rates and the helium-3 density in liquid helium.

\section{EXPERIMENTAL METHOD}

Suppose the decay volume is cylindrical with a length $L$ of $75 \mathrm{~cm}$ and a diameter $D$ of $7.5 \mathrm{~cm}$, i.e. the length to diameter ratio is $L / D=10$. The $\mathrm{CN}$ beam of $3 \mathrm{~cm}$ diameter passes the decay volume along the axis of the cylinder. The neutron decay rate in the volume is given as

$$
\dot{N}_{\beta}=\tau_{\beta}^{-1} \epsilon_{\beta} L \int_{A_{b}} d a \int_{v} d v \cdot I(v) \frac{1}{v}
$$

where $\tau_{\beta}$ is the neutron lifetime, $\epsilon_{\beta}$ is the detection efficiency of the beta decay in the given geometry, $A_{b}$ is the area of the beam, and $I(v)$ is cold neutron flux with respect to the neutron velocity $v$. 
When the CN beam passes through the decay volume, the ${ }^{3} \mathrm{He}$ nuclei in liquid helium capture neutrons via nuclear reaction $n+{ }^{3} \mathrm{He} \rightarrow p+t+764 \mathrm{keV}$. The capture rate is given as

$$
\dot{N}_{p+t}=\epsilon_{H e 3} \sigma_{H e 3}^{t h} v_{n}^{t h} n_{H e 3} L \int_{A_{b}} d a \int_{v} d v \cdot I(v) \frac{1}{v}
$$

The neutron lifetime $\tau_{\beta}$ can be obtained from the ratio of the neutron ${ }^{3} \mathrm{He}$ capture rate over the beta decay rate.

$$
\tau_{\beta}=\frac{\dot{N}_{p+t}}{\dot{N}_{\beta}} \cdot \frac{\epsilon_{\beta}}{\epsilon_{H e 3}} \cdot \frac{1}{\sigma_{H e 3}^{t h} v_{n}^{t h} n_{H e 3}}
$$

Eqn. (3) is the key expression in this experiment. It explicitly shows the measurement of $\tau_{\beta}$ is independent of the neutron flux as well as the geometry of the decay volume. The overall accuracy relies on that of the ratio of event rates $\kappa=\dot{N}_{p+t} / \dot{N}_{\beta}$, the helium-3 density $n_{H e 3}$ in liquid helium, and the detection efficiency of scintillation events $\epsilon_{H e 3}$ and $\epsilon_{\beta}$. The former two quantities will be experimentally acquired, and the detection efficiencies will be determined through simulations considering the calibration and background discrimination.

Here, we provide an estimate of count rates based on the published performance of the Fundamental Neutron Physics Beam Line (FnPB) in the Spallation Neutron Source (SNS) at Oak Ridge National Laboratory (ORNL), as shown in Fig. 1 [17. The neutron flux at $17 \pm 0.5 \AA$ is about $2.4 \times 10^{6} \mathrm{Ns}^{-1} \mathrm{~cm}^{-2} \mathrm{MW}^{-1} \mathrm{~s}^{-1}$. With a timeaveraged proton power of $1.8 \mathrm{MW}$ at $60 \mathrm{~Hz}$ of doublechopper, the incident rate of $16.5 \AA$ neutrons is about $3.1 \times 10^{7} \mathrm{~Hz}$. In this estimate, neutron lifetime is taken as the PDG suggested value $\tau_{\beta}=880 \mathrm{~s}$. It takes about $3 \mathrm{~ms}$ for the $16.5 \AA$ neutrons to pass $75 \mathrm{~cm}$ long decay volume, and the neutron decay rate is $3.55 \times 10^{-6}$ for a $\mathrm{CN}$ beam with a cross section of $3 \mathrm{~cm}$ diameter. There are an average of $108.5 \mathrm{~Hz}$ of neutron decay events. The natural abundance of ${ }^{3} \mathrm{He}$ in liquid helium is $X_{H е 3}=5 \times 10^{-7}$ in fractional concentration. Near isotopically pure ${ }^{4} \mathrm{He}$ with $X_{H e 3}<2.5 \times 10^{-13}$ has been produced as reported by McClintock. 18, Assuming liquid helium with $X_{\mathrm{He} 3}=$ $2 \times 10^{-10}$ can be prepared, an average of $489.3 \mathrm{~Hz}$ neutron capture events will occur simultaneously when the $\mathrm{CN}$ beam passes the decay volume. In reality, the neutron flux varies in time as to the source condition. The flux can be monitored by a neutron detector outside the beam exit window, and better in the form of a time-of-flight spectrum as in Fig. 1. It will be of great value for the systematic analysis.

\section{SCINTILLATION SIGNALS IN LIQUID HELIUM}

The number of beta decays and neutron captures will be counted via scintillation signals in liquid helium. Liquid

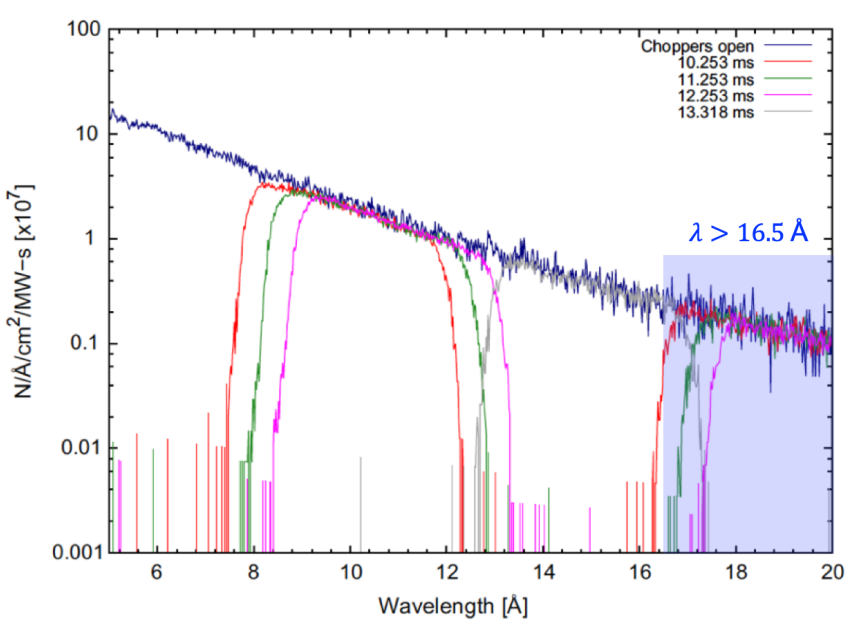

FIG. 1. Spectrum of cold neutron beam at the SNS FnPB beamline with choppers (a reprint of Figure 5 in Fomin 2015). The portion of wavelength $\lambda>16.5 \AA$ is highlighted in blue shadows.

helium is an ideal scintillator that has been proposed and studied for detection of neutrino [19] and dark matter [20, 21]. Compared to those experiments, the detection volume in the current beam lifetime experiment is very confined. High detection efficiency of scintillation photons can be practically achieved, as well as a wide energy window of high resolution and small lower bound.

In a neutron beta decay event, scintillation is generated by the electron recoil, as the counterpart proton is too slow. About $35 \%$ of the total energy in each electron recoil above $1 \mathrm{keV}$ creates $\mathrm{He}_{2}^{*}$ molecules of excited singlet state $\mathrm{He}_{2}\left(A^{1} \Sigma_{u}^{+}\right)$in liquid helium. The singlets radiatively decay in less than $10 \mathrm{~ns}$ and emit about 22 extreme ultraviolet (EUV) photons per $\mathrm{keV}$ of recoil energy, with a spectrum spanning from $13 \mathrm{eV}$ to $20 \mathrm{eV}$ and centering at $16 \mathrm{eV}$. 22] It forms the prompt pulse of scintillation light. There are about $1.7 \times 10^{4}$ photons per beta event at the end point energy of $782 \mathrm{keV}$ in the neutron beta decay spectrum. On the other hand, the neutron capture is purely a nuclear recoil event, the scintillation process of which is similar but of different features. About $13 \%$ of the total energy of $764 \mathrm{keV}$ converts into a prompt light pulse and results in about $6.4 \times 10^{3}$ photons per capture event. 23] The stopping power $d E / d x$ for a nuclear recoil in superfluid liquid helium of a density $\rho=0.145 \mathrm{~g} \mathrm{~cm}^{-3}$

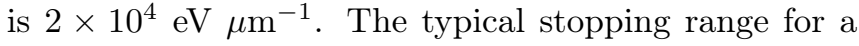
$800 \mathrm{keV}$ nuclear recoil is $40 \mu \mathrm{m}$. By contrast, the stopping power for an electron recoil is only $40 \mathrm{eV} \mu \mathrm{m}^{-1}$ [24] on average for a $800 \mathrm{keV}$ electron recoil, and its stopping range can reach up to $2 \mathrm{~cm}$. Therefore, a diameter of $7.5 \mathrm{~cm}$ is sufficient to prevent almost all of the electron recoils born in the $3 \mathrm{~cm}$ diameter $\mathrm{CN}$ beam from touching the inner surface of the decay volume. It nearly guarantees no quenching of prompt scintillation on the wall. Because of dramatic difference in the track length, the scintillation light of decay events is much more dispersed spatially 


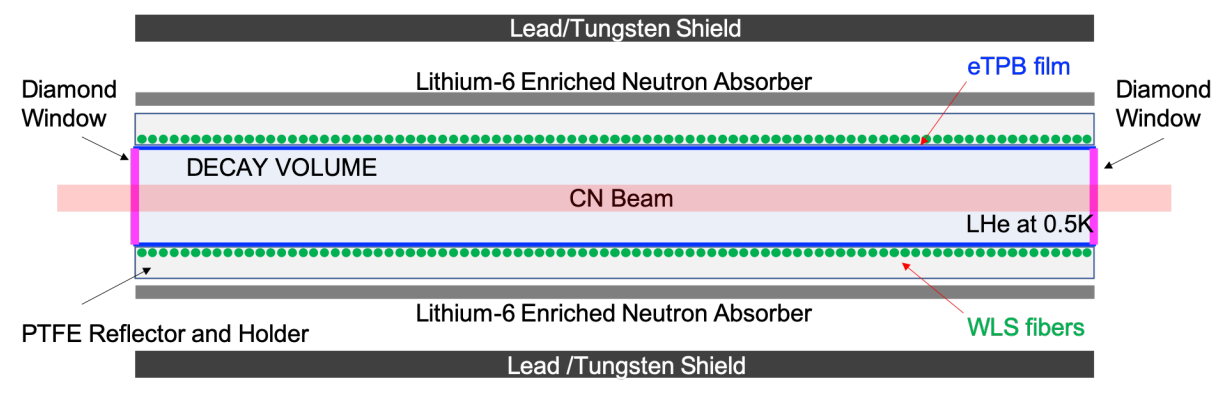

FIG. 2. Schematic of the conceptual detector (non-scaled)

than that of capture events. The former appears as a line of chained point sources, whereas the latter as a single point source.

In addition, both electron and nuclear recoils also generate a large amount of triplet $\mathrm{He}_{2}^{*}$ excimers $\left(a^{3} \Sigma_{u}^{+}\right)$, which has a $13 \mathrm{~s}$ lifetime in liquid helium. The radiative decay of the triplet excimers is forbidden as it requires a spin flip; yet it can occur via the bimolecular Penning ionization that converts a portion of the triplet into singlet, most likely in a high density of triplet excimers along the recoil track. This type of scintillation light appears as temporally scattered after-pulses of single EUV photons, whose occurrence rate decreases as to a combination of two components dependent exponentially and inversely on time, respectively, $f(t)=A e^{-t / \tau_{s}}+B / t+C$. It has been experimentally demonstrated that the $1 / t$ component of the electron recoils is much weaker than that of the nuclear recoils. 25. This feature is useful to distinguish the decay events from the capture events.

\section{DETECTION OF SCINTILLATION}

A standard method has been well developed to detect the EUV scintillation in liquid helium by many experiments. 22, 23, 25, 27] Based on the known technologies, we describe a conceptual design as a baseline for a quantitative analysis. The EUV scintillation light is first converted into a blue spectrum near $400 \mathrm{~nm}$ by an organic fluor - tetraphenyl butadiene (TBP). A thin layer of evaporated TPB (eTPB) can be coated on an acrylic film and wrapped into a cylinder as the boundary of the decay volume. The eTPB coating faces the inside of the decay volume. Optical fibers can be molded with a structural support as if wound on the outside of the film cylinder to collect light. Wavelength shifting (WLS) fibers are a common option to convert the emitted blue light into a green spectrum near $500 \mathrm{~nm}$ along with a redistribution of photon phase space. A portion of the shifted light can be trapped inside the fiber by total internal reflection and transmitted to the photon sensors. A schematic of a conceptual detector is shown in Fig. 2, It is similar to the light collection system used in the storage neutron lifetime with UCNs in liquid helium filled magnetic trap
[26], but in this setup, the eTPB film is installed inside of the fibers and the fibers will have a nearly full coverage of solid angle to collect light.

The overall light conversion efficiency is estimated as follows. Owing to the large $L / D$ ratio of the decay volume, more than $96 \%$ in solid angle of the scintillation light can be converted by TPB for the events occurring in the central region, as shown in Fig. 3. The conversion efficiency of eTPB has been demonstrated to be greater than unity. 28. Since a thick eTPB coating often appear opaque for visible light due to its surface roughness, the blue photons heading inwards the decay volume might reflect and diffuse on the eTPB coating, which makes it hard to judge how much can actually penetrate through and be collected by WLS fibers on the far side. Hence, as a moderate estimate, we only take into account the $50 \%$ of eTPB re-emitted blue photons that travel outwards to the adjacent WLS fibers. Approximately $90 \%$ of them can impinge on the fiber cores with the help of a PTFE reflector, which is also a structural holder clamped on the outside, and then about $80 \%$ is absorbed and shifted into green light. The double cladding WLS fibers made by Kuraray have a trapping efficiency of $5.4 \%$ in one direction. 29] When read on both ends, $10.8 \%$ of the shifted green light can be conveyed towards the 2 photon sensors. Since the fiber has a bending loss of about $4 \%$ per turn on a $7.5 \mathrm{~cm}$ diameter curve and an attenuation length longer than $7.5 \mathrm{~m}$, the fiber length must be constrained. In each detector unit, a round WLS fiber of $1 \mathrm{~m}$ long and $1 \mathrm{~mm}$ diameter is wound around the decay volume by 3 turns, and two photon sensors read at both of its ends. To cover the whole length of the decay volume, it needs 250 units. The average transmission efficiency of such a configuration is about $90 \%$ along the fiber. With regard to the difficulty of making large amount of superfluidleak-tight fiber feedthroughs, there must be two optical breaks at the windows of the liquid helium vessel, each of which has a $90 \%$ transmission. As for the photon sensor, we may employ silicon photomultipliers, which have a typical quantum efficiency of $34 \%$ for the versions with a large microcell size. [30] The overall conversion efficiency $\eta_{\text {tot }}$ is $0.9 \%$, i.e. an average of 9 photo-electrons $(P E)$ can be detected per $1 \times 10^{3}$ EUV scintillation photons. The mean signals for beta events of the spectrum peak 
energy at $245 \mathrm{keV}$ and the endpoint energy at $782 \mathrm{keV}$ are $\bar{N}_{P E}^{(p e a k)}=49.8$ and $\bar{N}_{P E}^{(e n d p t)}=158.9$, respectively. The energy resolution $\Delta E_{P E}$ is about $5 \mathrm{keV}$ per $\mathrm{PE}$. On the other hand, the signals for the neutron capture events of recoil energy at $764 \mathrm{keV}$ is $\bar{N}_{P E}^{(p+t)}=57.6$. It coincides with beta events of $283.8 \mathrm{keV}$, close to the peak of the beta spectrum.

\section{DETECTOR RESPONSE AND EVENT RECONSTRUCTION}

We perform a preliminary study on the response of detectors by Monte Carlo simulations. As listed below, several assumptions have been adopted to simplify the model but present the essential physics as a proof of principle. Further modelling with more details is needed.

(i). Only the prompt scintillation signals are recorded for all the events. It means the decay and capture events cannot be distinguished among the simulated data. Yet in real experiment, they might be distinguishable by the difference in the $1 / t$ timedependent occurrence rates of the after-pulses.

(ii). The scintillation light for the capture events is emitted from a point source as their track length is tens of microns, whereas that for the beta events is from an energy-dependent straight tracks of length up to $2 \mathrm{~cm}$. For electron recoils, more energy deposits in the vicinity of the track end as it slows down. The spatial energy deposition approximately follows $d E / d r \propto r^{2}$, where $r$ is the geometric distance from the starting point of electron recoil. 31] Further studies can be performed on simulated scattering tracks with productions of secondary $\delta$-electrons.

(iii). Only the outward-going portion of the eTPB converted light can be collected by the adjacent fibers, but none of the inward-going, as the latter reflects and diffuses on the coating into a broader distribution over all the detectors, yet much weaker than the former.

(iv). The EUV light converted by eTPB will be collected by the fibers tightly wound against the thin film at the same axial position, i.e. the solid angle of light from an event projecting on the section of the eTPB film is equivalent to that on the detector lying against the film.

(v). All the detectors have the same efficiency. In reality, the efficiency of detectors are different and may vary with time. Calibrations are necessary and will be discussed in Subsection VC.

(vi). There is no time information in this simulation. We assume all the events are in the coincident time window as the cold neutron beam passes the decay volume. Timing information will greatly improve the position reconstruction and be a practical way of minimizing background.

A sampling on starting position $\vec{x}_{0}$ of events as to a uniform distribution function, $\operatorname{Pr}\left(\vec{x}_{0}\right)=$ const., is carried out in the beam-occupied volume. $4 \times 10^{7}$ random events are generated and assigned as either capture or decay according to a preset ratio of 4.498. The capture events are of a point source in the beam-occupied volume, whereas the decay events are of a straight line source that may extend out. For each event, emission of scintillation light is isotropic, and the portion of solid angle received by each and every of the 250 detectors is simulated as the hit probability of each detector given the source position $\vec{x}$, $\operatorname{Pr}\left(n_{\text {det }} \mid \vec{x}\right)$, where $n_{\text {det }} \in[1,250]$ is the detector number. Fig. 3 plots the accepted portion of solid angle by one detector, e.g. at $z=-1.65 \mathrm{~cm}$, and sum of that over all the detectors for each event at various axial positions $z$, respectively. The events ending in the beam path are highlighted in blue. It shows the total accepted solid angle has a weak dependence on the radial ending position of the event. With all the detectors functioning, it can cover more than $96 \%$ of solid angle for events in a central region spanning $42 \mathrm{~cm}$ in the axial direction, as shown by the upper plot in Fig. 3. The events lying within the axial edges of a unit detector have about $4 \%$ of chance to be registered by this detector, as shown in the lower plot in Fig. 3. The overall detector hit probability $\operatorname{Pr}\left(n_{\text {det }}\right)$ is derived by the integral over the entire volume $V_{0}, \operatorname{Pr}\left(n_{\text {det }}\right)=\int_{V_{0}} d \vec{x} \operatorname{Pr}\left(n_{\text {det }} \mid \vec{x}\right) \operatorname{Pr}(\vec{x})$, and plotted in the Fig. 4. For detectors in the central region, the overall hit probability is about $0.40 \%$ for scintillation light of an event at any position to be registered; while for those close to the ends, the chance is naturally much less.

\section{A. Reconstruction of Event Position}

With the registered photon numbers $N_{P E}\left(n_{\text {det }}\right)$ from an event on a series of detectors $n_{\text {det }}, 1 \leqslant n_{\text {det }} \leqslant 250$, the probability of event position within a given sub-volume $\Delta V\left(z_{k}\right)$ centered at $z_{k}$ is derived in Eqn. (4) by the Bayes' theorem. It is on the assumption that every detector is independent, i.e. no cross talks. The probability of an event within a certain region of interest, e.g. the $42 \mathrm{~cm}$ long central region, can be calculated as the accumulated probability, $\sum_{z_{k}} \operatorname{Pr}\left(\Delta V\left(z_{k}\right) \mid N_{P E}\left(n_{\text {det }}\right)\right)$. Fig. 5 shows an example of the simulated electron recoil event with 77 observed $P E$ s distributed on several detectors. The entire volume $V_{0}$ is divided into sub-volumes $\Delta V\left(z_{k}\right)$ as disks of $1 \mathrm{~cm}$ thick, and the distribution probability of event position for each sub-volume is calculated and plotted in Fig. 5. The accumulated probability for this event to occur within the $42 \mathrm{~cm}$ long central region is $93.7 \%$. In general, the more $P E$ s observed, the more accurate the reconstructed position of the event. However, since the events near the ends of the decay volume lose a significant portion of scintillation light on the end windows, 


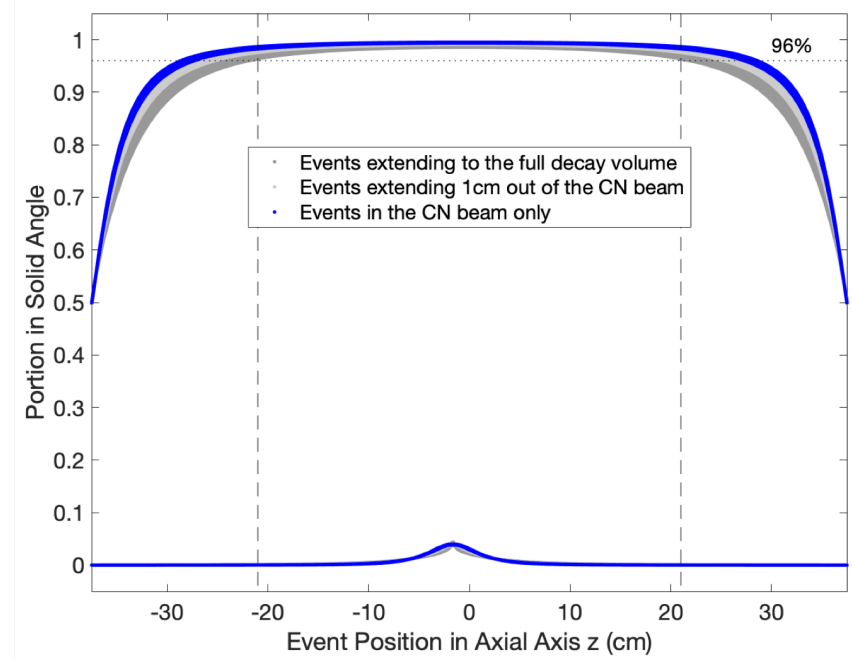

FIG. 3. The coverage of solid angle for events ending at different axial positions in the decay volume. The upper plot is the total coverage of solid angle by the sum of all the detectors; and the lower plot is that by one of the central detectors.

i.e. information is truncated, the reconstructed positions are biased towards the center. Therefore, the accumu- lated probability of positions inside the central region of $42 \mathrm{~cm}$ is a better fiducial cut in identifying events thereof than that of reconstructed positions within the same region, especially for low $P E$ events. A combination of the accumulated probability and reconstructed positions is a good way to select events in a region with relatively identical position distribution and uniform ratio of capture-to-decay event rates. It is demonstrated in Fig. 6, where the events are selected by a combination of the following criteria, (a). reconstructed positions within $\pm 15 \mathrm{~cm}$ and (b). accumulated probability of more than $80 \%$ inside the central region of $42 \mathrm{~cm}$. The number of selected events is about $42 \%$ of the total. The spectrum of the selected events with respect to the $P E$ number is plotted in Fig. 7. The capture events overlap with the decay events in the mid range and the spectrum cuts off at a lower bound of $4 P E \mathrm{~s}$, equivalent to $K E_{e}$ of $20 \mathrm{keV}$, on purpose to exclude random few-photon backgrounds. Both the low and high $P E$ events have a relatively significant position uncertainty due to few $P E$ s and long tracks, respectively. Therefore, the fiducial cut above inevitably introduces a detection inefficiency, $1-\epsilon_{c u t}\left(N_{P E}\right)$, and hence, a distortion of spectrum. Such an effect is extracted and fitted as in Fig. 8, and then included in the fit function, Eqn. (7), in the following Subsection VB.

$$
\operatorname{Pr}\left(\Delta V\left(z_{k}\right) \mid N_{P E}\left(n_{d e t}\right), 1 \leq n_{d e t} \leq 250\right)=\int_{\Delta V\left(z_{k}\right)} d \vec{x} \frac{\sum_{n_{d e t}=1}^{250} N_{P E}\left(n_{d e t}\right) \operatorname{Pr}\left(n_{d e t} \mid \vec{x}\right) \operatorname{Pr}(\vec{x})}{\sum_{n_{d e t}=1}^{250} N_{P E}\left(n_{d e t}\right) \operatorname{Pr}\left(n_{d e t}\right)}
$$

\section{B. Determination on the Ratio of Capture-to-Decay Event Rates}

In order to resolve the ratio of capture-to-decay event rates from the acquired spectrum, a theoretical model for fitting is constructed. It consists of three components: the neutron decay spectrum, the single capture peak and the background. In this study, we only simulate signals of the former two, but omit the effect of the background, because it will be poorly defined without the knowledge of the actual system. Some discussions on the possible backgrounds will be presented in Section VI. The neutron beta decay spectrum is formulated as

$$
\begin{gathered}
\frac{d \Gamma}{d E_{e}}=W\left(E_{e}\right) \propto F_{n}\left(E_{e}\right) p E_{e}\left(E^{0}-E_{e}\right)^{2} \\
F_{n}\left(E_{e}\right)=4 \exp \left(\pi \alpha E_{e} / p\right) \frac{\left|\Gamma\left(1-i \alpha E_{e} / p\right)\right|^{2}}{\Gamma(3)^{2}}
\end{gathered}
$$

where $\alpha=1 / 137$ is the fine structure constant, $E^{0}$ is the endpoint energy, $E_{e}=K E_{e}+m_{e}$ is the total electron energy, $m_{e}$ is the electron mass, $K E_{e}$ is the electron kinetic energy, $p=\left(E_{e}^{2}-m_{e}^{2}\right)^{1 / 2}$ is the momentum of electron, and $F_{n}\left(E_{e}\right)$ is the Fermi function for neutrons defined as Eqn. (6).

$$
\begin{aligned}
f\left(N_{P E}\right)= & \frac{1}{1+\tilde{\kappa}} \frac{\epsilon_{c u t}\left(N_{P E}\right)}{C_{e}} \sum_{M_{P E}} \operatorname{Pois}\left[N_{P E}, \Lambda_{e}\left(M_{P E}\right)\right] \\
& +\frac{\tilde{\kappa}}{1+\tilde{\kappa}} \frac{1}{C_{p+t}} \operatorname{Pois}\left[N_{P E}, \lambda_{p+t}\right]+b \\
\Lambda_{e}\left(M_{P E}\right)= & \int_{\left(M_{P E}-0.5\right) / \eta_{e} \Omega\left(M_{P E}\right)}^{\left(M_{P E}+0.5\right) / \eta_{e} \Omega\left(M_{P E}\right)} \frac{d \Gamma}{d E_{e}} d E_{e}
\end{aligned}
$$

The entire spectrum contains a Poisson smearing due to the random process in the light transportation and conversion into PEs. Though the deposited energy from neutron capture events is single-valued at $764 \mathrm{keV}$, it appears as a much broadened peak. The probability density function for this spectrum $f\left(N_{P E}\right)$ is expressed by Eqn. (7), where $N_{P E}$ is the total $P E$ number collected by all the detectors, $\epsilon_{\text {cut }}\left(N_{P E}\right)$ is efficiency of detection related to the fiducial cut, and $C_{e}$ and $C_{p+t}$ are the 


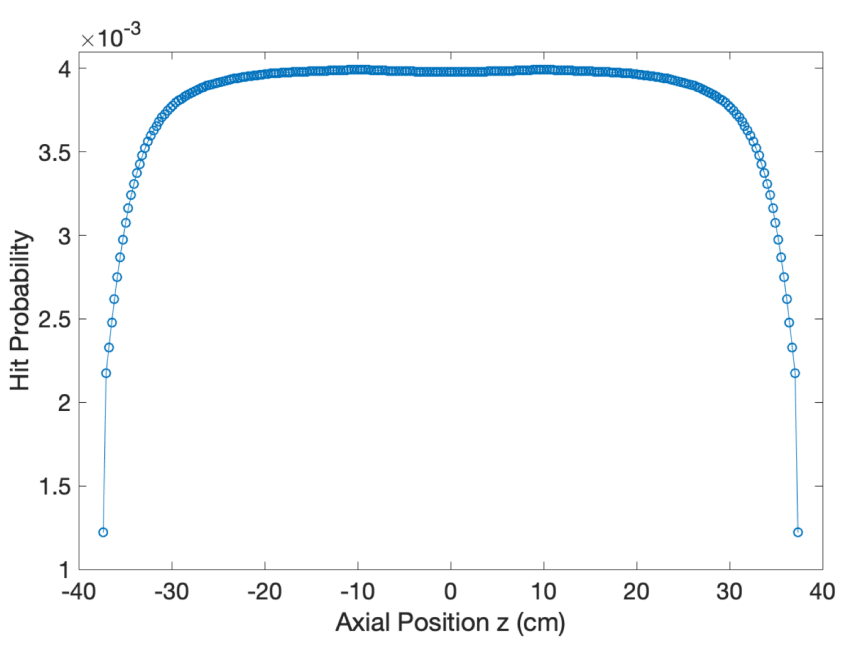

FIG. 4. The overall hit probability on each of the 250 detectors
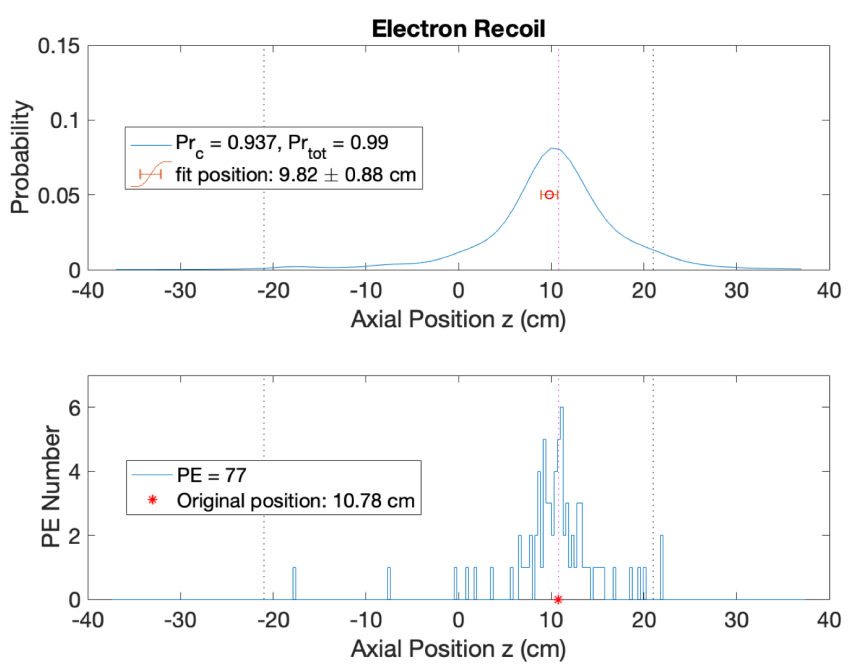

FIG. 5. An example on the deduced possibility of event axial position based on the observed distribution of $77 \mathrm{PEs}$.

normalization factors for the spectrum bins above the lower bound of $4 P E \mathrm{~s}$. There are 3 fit parameters: $\eta_{e}$ is the conversion rate of $K E$ to $P E$ s for electron recoils, $\lambda_{p+t}$ is the mean $P E$ number for the capture events, and most importantly, $\kappa=\Delta N_{p+t} / \Delta N_{\beta}$ is the ratio of captureto-decay event rates. The Maximum Likelihood (ML) method is employed to acquire the best fit and associated errors of the above 3 parameters. $\kappa$ in Eqn. (7) is marked as $\tilde{\kappa}$, as the fitting only acquires the capture-to-decay ratio within the data bins. The complete $\kappa$ is obtained by applying a correction to $\tilde{\kappa}$ to include the detection efficiencies $\epsilon_{\mathrm{He} 3}=1$ and $\epsilon_{\beta}=0.9878$ due to a missing spectrum of events less than $4 P E$ s. The corrected result is listed in Table I] and the plots in Fig. 7 show the result of the ML fit (in dotted line) on top of the acquired spectrum (in solid line) with the fiducial cut as stated in Subsection $\mathrm{VA}$. It shows the fitting can extract $\kappa$ value
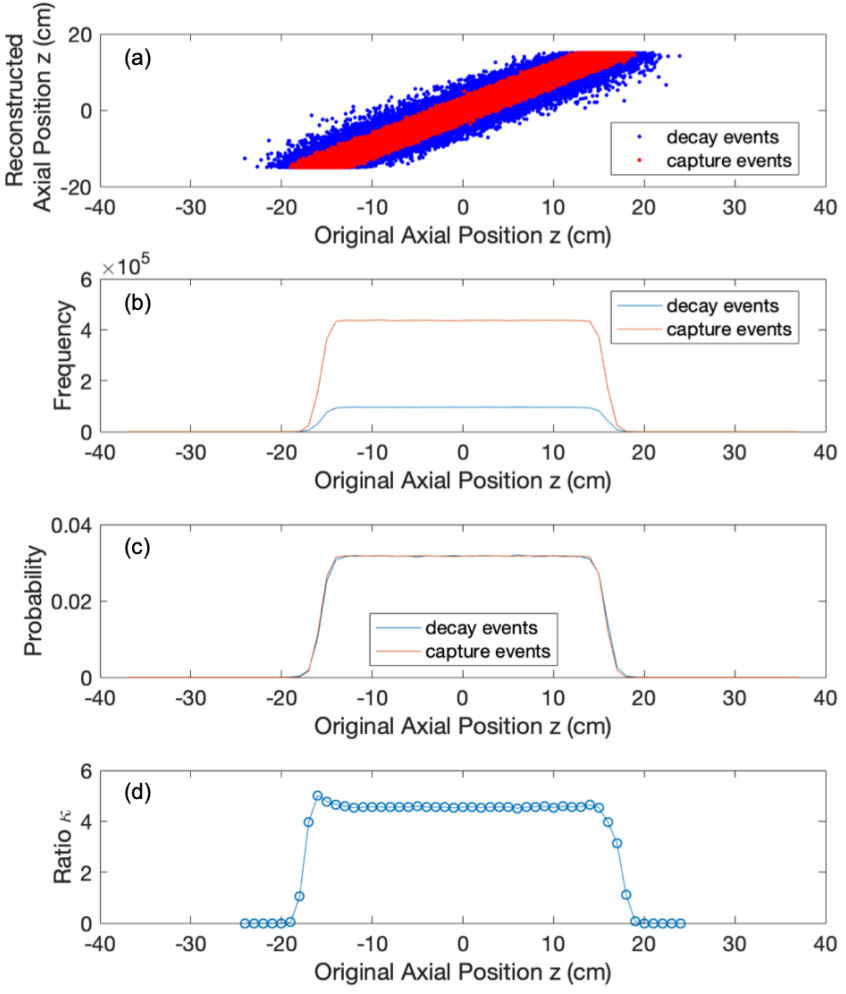

FIG. 6. Analysis of the combined fiducial cut on the simulated data. (a). the reconstructed axial position $z$ of both the simulated decay and capture events plotted against their original position; (b) and (c). frequency and probability distribution of the selected decay and capture events by the fiducial cut as to their original positions, respectively; (d). the ratio $\kappa$ calculated within each bin of original position $z$.

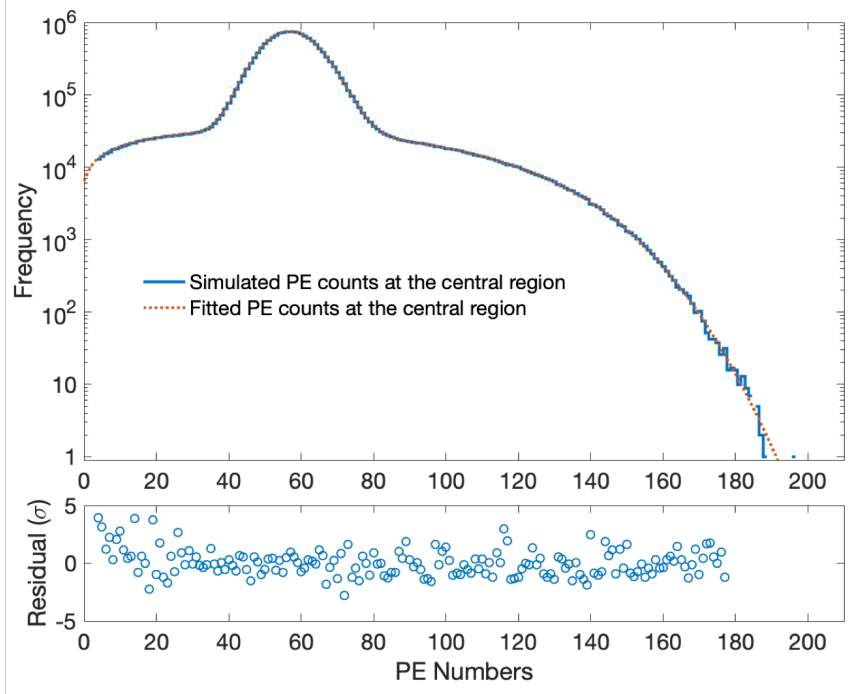

FIG. 7. Simulated neutron beta decay spectrum in addition to the neutron capture peak in solid line and the result of the ML fitting in dotted line. The residual of fitting is shown in the lower plot. $\chi^{2} / n d f=1.4$ 
TABLE I. Result of ML fit to the simulated spectrum

\begin{tabular}{lccc}
\hline & $\kappa=\Delta N_{p+t} / \Delta N_{\beta}$ & $\eta_{e}$ & $\lambda_{p+t}$ \\
\hline Preset Value & 4.4975 & 0.2032 & 57.254 \\
Monte Carlo & 4.4997 & 0.2032 & 57.292 \\
ML Fit & $4.4983 \pm 0.0040$ & $0.2037 \pm 0.0001$ & $57.291 \pm 0.002$ \\
\hline
\end{tabular}

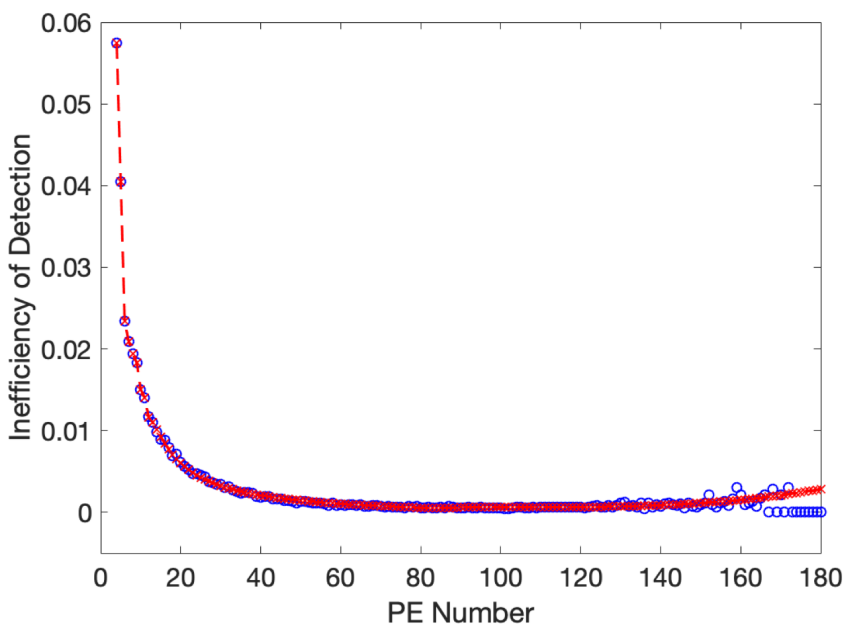

FIG. 8. Inefficiency of detection, $1-\epsilon_{c u t}\left(N_{P E}\right)$, related to the combined fiducial cut of reconstructed positions within $\pm 15 \mathrm{~cm}$ and $80 \%$ accumulated probability within $\pm 21 \mathrm{~cm}$. The simulation data is plotted in circles, and the piece-wise fit is plotted in dashed line.

at an accuracy well within $0.1 \%$.

\section{Reconstruction of Event Energy and Detector Calibration}

Reconstruction of event energy will be performed in calibration of all the detectors as a cross reference. In the previous subsection, the conversion efficiency $\eta_{e}$ for electron recoils is fitted through the analysis of the neutron decay spectrum, and the reconstructed energy is obtained as $E_{e}=N_{P E} / \eta_{e}$. In reality, each photon sensor has a different quantum efficiency $\eta_{S i P M}^{(i)}$, and each fiber has a variation in transmission efficiency $\eta_{f i b e r}^{(i)}$. The overall detection coefficient $\eta_{t o t}(\vec{x})$ thus varies for different sub-volumes, due to the variation of solid angles upon detectors of different quantum efficiency. It is a common approach to use conversion electron sources, such as ${ }^{109} \mathrm{Cd}$ $(63,84 \mathrm{keV}),{ }^{139} \mathrm{Ce}(127,160 \mathrm{keV}),{ }^{113} \mathrm{Sn}(364,388 \mathrm{keV})$, ${ }^{207} \mathrm{Bi}(481,975,1047 \mathrm{keV})$ for calibrations in between production runs. 35] These sources can be placed in many designated positions to map out the response of different detectors. During the production runs, the calibration can also be done with the neutron capture peak, and additional deposits of $\alpha$ source or lithium neutron capture film on the end windows of the decay volume.

\section{BACKGROUND SUPPRESSION}

In order to achieve a highly accurate measurement on the ratio of capture-to-decay rates, background signals must be properly suppressed, discriminated or subtracted. Cosmic ray muons can be easily identified by coincidence in the veto detectors surrounding the apparatus. The static radioactive backgrounds from materials of the apparatus can be shielded by a thick layer of lead or tungsten as shown in Fig. 2 and characterized in the background runs. The gamma rays from the cold neutron source can be greatly suppressed by bending the beam direction out of sight with proper neutron optics. 17] The most harmful type of backgrounds are the gamma rays produced by the neutron-induced activation near the decay volume and undergoing Compton scattering on liquid helium inside the decay volume and the surrounding WLS fibers. The Compton electrons are identical to the decay events on the features of scintillation signals. It hence will be pooled in the $P E$ spectrum and modelled as $b$ in Eqn. 77). The spectrum of the Compton electrons produced by gamma rays above $4 \mathrm{MeV}$ is mostly flat in the region of neutron decay spectrum. Some of the delayed gamma rays can be characterized during the intervals between the $\mathrm{CN}$ beam pulses, such as the $1.6 \mathrm{MeV}$ gamma rays emitted at a half-life of $11.16 \mathrm{~s}$ from the neutron activated fluorine. In this paper, we focus on two types of prompt gamma rays due to the neutron captures by the window material and by hydrogen, $p+n \rightarrow d+\gamma(2.2 \mathrm{MeV})$, in the plastic components surrounding the decay volume, such as the WLS fibers, etc. They are believed to be the major contributors to the backgrounds.

The first measure to suppress neutron-induced gamma rays is to reduce the capture and scattering of neutrons on the window materials. Polycrystalline CVD diamond is a good option, because carbon has relative small scattering and capture cross sections, and a thin window of $5 \mathrm{~cm}$ diameter and $1 \mathrm{~mm}$ thickness [34] is commercially available with a good mechanical strength. The capture cross section of carbon for the $16.5 \AA$ cold neutrons is 0.032 barns and the capture fraction is $5.66 \times 10^{-4}$. For a cold neutron flux of $3.1 \times 10^{7} \mathrm{CN} \mathrm{s}^{-1}$, about $1.73 \times 10^{4} \mathrm{~Hz}$ of neutrons are captured with an emission of prompt gamma rays mostly at energies of $1.3,3.7$ and $4.9 \mathrm{MeV}$. A simulation shows the intensive prompt gamma rays result in more than $1200 \mathrm{~Hz}$ Compton events in liquid helium inside the decay volume, and more than $200 \mathrm{~Hz}$ in the polystyrene WLS fibers. Most events distribute spatially near the windows, and temporally at the moments when the neutron flux passes the windows. Although the number of the window-originated Compton events greatly overwhelms that of the decay events, they can be separated in time if the neutron beam can be chopped into sharp pulses both in time and in energy. The neutron decays will then appear in the time sequence as scattered single events of electron recoil in between two intensive bursts of Compton events when neutrons pass the entrance and exit windows, respectively. Since only the events in the central region 
of the decay volume are pooled to construct the energy spectrum, a proper time cut could be effective to eliminate impacts of the window-originated Compton events. In return, the bright bursts of Compton events can be used as a calibration reference of the beam flux.

The scattering of cold neutrons on the windows at $0.5 \mathrm{~K}$ is dominated by incoherent scattering, which is an s-wave scattering independent of the incident velocity. The incoherent cross section of carbon for the $16.5 \AA$ cold neutrons is 0.001 barns, and the scattered fraction is $1.76 \times 10^{-5}$. For the same cold neutron flux as above, about $540 \mathrm{~Hz}$ of neutrons are scattered isotropically from both the windows into the delay volume and interact with hydrogens on the fibers. The incoherent scattering cross section of hydrogen is 80.26 barns, much larger than the capture cross section of 3.05 barns for $16.5 \AA$ cold neutrons. The stray neutrons will mostly scatter incoherently in the plastics and has a small chance of being captured by the hydrogen nuclei. In order to minimize the chance of captures, the second measure is to deploy an effective neutron absorber on the outside of the plastics so as to capture all the outgoing stray neutrons. Lithium-6 enriched material is an ideal option, since lithium- 6 has a large neutron absorption cross section of 646.9 barns for $16.5 \AA$ cold neutrons, and there is no associated emission of gamma rays in the reaction, ${ }^{6} L i+n \rightarrow \alpha+t+4.78 \mathrm{MeV}$.

A simulation on neutron scattering and capture is carried out on the geometry of a $1 \mathrm{~mm}$ thick polystyrene fibers around the decay volume, a $5 \mathrm{~mm}$ thick PTFE holder clamped on the fibers, and a sufficiently thick lithium absorber at the outermost shell that absorbs all the stray neutrons, as illustrated in Fig. 2. It is found about $4.3 \%$ of the scattered neutrons are captured by the fibers and $0.4 \%$ by the PTFE holder. The neutron captures on hydrogen in the fibers do not induce any significant scintillation in the polystyrene, as the kinetic energy of deuterium is merely about $1.3 \mathrm{keV}$. The resultant prompt gamma rays of $2.2 \mathrm{MeV}$ contribute a background of Compton events at $0.56 \mathrm{~Hz}$ in liquid helium inside the decay volume, and $0.62 \mathrm{~Hz}$ in the polystyrene WLS fibers. As shown in Fig. 9, the Compton events have a higher chance to occur near the windows. Since the majority of Compton electrons are at energies near the Compton peak of $2.0 \mathrm{MeV}$, it adds about $0.05 \%$ to the total counts of neutron decay events with a fiducial cut of $\pm 15 \mathrm{~cm}$ on the central region. It can be characterized and corrected in data analysis.

\section{DETECTION OF ${ }^{3}$ HE DENSITY WITH UCNS}

The last quantity crucial to determine the neutron lifetime in Eqn. (3) is the ${ }^{3} \mathrm{He}$ density $n_{H e 3}=2.18 \times 10^{22} X_{H e 3}$, where the fractional concentration $X_{H e 3}$ of about $2 \times 10^{-10}$ needs to be prepared and characterized in high precision. It has been demonstrated that Atom Trap Trace Analysis method can measure the abundance of rare isotope ${ }^{39} \mathrm{Ar}$

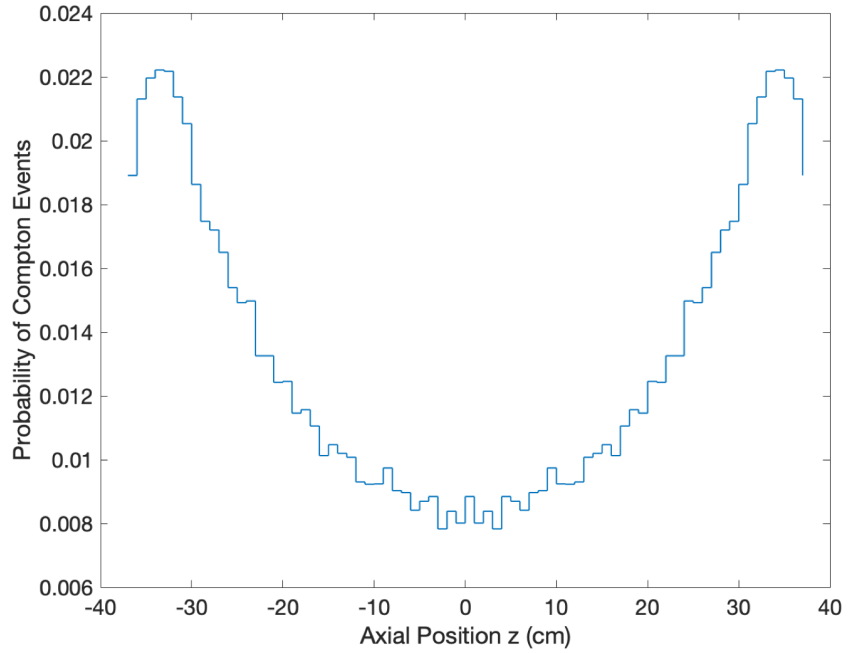

FIG. 9. Probability of Compton electron events in the axial axis of the decay volume induced by the prompt gamma rays due to hydrogen-captures of neutrons scattered from the beam inlet and outlet diamond windows.

at the isotopic abundance level of $10^{-16}$. 36 Similar technology may also be developed for detection of the ${ }^{3} \mathrm{He}$ abundance at the sub- $0.1 \%$ precision. In this paper, we provide another possible method: measuring the time-dependent neutron capture rate in the sample liquid helium via scintillation. The neutrons involved in the ${ }^{3} \mathrm{He}$ capture interaction are not cold neutrons but rather ultracold neutrons (UCN), which are stored in a material bottle of known storage time filled with the sample liquid helium. Because UCNs will be uniformly distributed in the storage volume and some scintillation will quench on the walls, it is difficult to resolve a well-defined spectrum as in Subsection VB.

\section{A. UCN Storage in a Neutron-Friendly Volume}

Suppose the sample liquid helium fills a storage volume made of UV transmitting acrylic tube of $75 \mathrm{~cm}$ long, $7 \mathrm{~cm}$ ID and $7.5 \mathrm{~cm}$ OD. It is sealed at both ends and coated with deuterated film on the inside so that it is hermetic and friendly to UCNs. It can be installed inside the detector setup similar to that used in the beam lifetime measurement, except the PTFE reflector can be as thin as $50 \mu \mathrm{m}$ because of the following two reasons: $(\mathrm{A})$. the structural support can be loaded to the storage tube; and (B). PTFE generates a high level of background due to the delayed gamma rays from neutron-activated fluorine as discussed in Subsection VIIE, The UCN storage follows

$$
\dot{N}_{U C N}(t)=-\frac{N_{U C N}(t)}{\tau_{t o t}}
$$




$$
N_{U C N}(t)=N_{0} \exp \left(-\frac{t}{\tau_{t o t}}\right)
$$

where $N_{0}$ is the initial number of UCN and $\tau_{t o t}$ is the storage time constant.

Several factors contribute to the storage time constant of this volume,

$$
\tau_{\text {tot }}^{-1}=\tau_{H e 3}^{-1}+\tau_{\beta}^{-1}+\tau_{\text {up }}^{-1}+\tau_{\text {loss }}^{-1}
$$

$\tau_{H e 3}$ is the neutron ${ }^{3} \mathrm{He}$ absorption time constant of interest as given in Eqn. (11). For $X_{\mathrm{He} 3}=2 \times 10^{-10}$, $\tau_{\text {Heз }}$ is about $195.2 \mathrm{~s}$, which dominates the total storage time, compared to the neutron lifetime $\tau_{\beta} \approx 880 \mathrm{~s}$.

$$
\frac{1}{\tau_{H e 3}}=n_{H e 3} \sigma_{H e 3}^{t h} v_{H e 3}^{t h}=2.56 \times 10^{7} X_{H e 3} \mathrm{~s}^{-1}
$$

UCNs suffer a loss from captures or up-scattering of the wall nuclei. Such an effect can be described by Schrödinger equations with one-dimensional potential and characterized by a loss probability per bounce, $f\left(E_{U C N}\right)$. The rate of wall collisions is given by $(v A / 4 V)$, where $v$ is the UCN velocity, $A$ is the area of the storage chamber, and $V$ is its volume. The contribution of wall losses to the storage time is then given as

$$
\frac{1}{\tau_{\text {wall }}}=f\left(E_{U C N}\right)\left(\frac{v A}{4 V}\right)
$$

Generally, the hydrogen in the organic materials has a large up-scattering cross section for the UCNs. Therefore the hydrogen in contact with UCN must be replaced with deuterium. The inner wall of the storage volume needs to be coated with a layer of deuterated polystyrene (dPS), whose Fermi potential is about $160 \mathrm{neV}$. The TPB converter coating also needs to be deuterated. These technologies are under development and tests by the SNS nEDM collaboration. 37 With a loss probability per bounce of $f\left(E_{U C N}\right)=10^{-5}$, the same requirement as the SNS nEDM UCN storage cells [38, the time constant due to wall loss $\tau_{\text {wall }}$ is about $1672.0 \mathrm{~s}$.

$\tau_{u p}$ is the loss rate due to upscattering of neutrons by quasi-particles, phonons and rotons, in superfluid helium. It is greatly suppressed by a Boltzmann factor. At $T<$ $0.6 \mathrm{~K}$, the dominant process is multi-phonon scattering following

$$
\frac{1}{\tau_{u p}}=\frac{[T(K)]^{7}}{100 \mathrm{~s}}
$$

At $T=0.5 \mathrm{~K}, \tau_{u p}=12800 \mathrm{~s}$. With all the contributions above included in Eqn. 10, the storage time constant $\tau_{\text {tot }}$ is about $144.2 \mathrm{~s}$.

\section{B. UCN Production}

The UCNs for this measurement are produced in situ in the neutron decay volume by super-thermal process: a $8.9 \AA$ beam of $\mathrm{CNs}$ are down-scattered inelastically into UCNs via exciting a single phonon in superfluid helium. 32 The UCN density can build up in the decay volume with the time constant $\tau_{\text {tot }}$. The accumulated UCN density in liquid helium exposed to the CN beam is given by

$$
\rho_{U C N}\left(t_{\text {fill }}\right)=R \tau_{\text {tot }}\left[1-\exp \left(-\frac{t_{\text {fill }}}{\tau_{\text {tot }}}\right)\right]
$$

where $\tau_{\text {tot }}$ is the storage time constant of the UCN as given in Eqn. $(10)$, and the production rate per unit volume $R$ is given by

$$
R=2.2 \times 10^{-8}\left(\frac{d \Phi}{d E}\right) \mathrm{cm}^{-3} \mathrm{~s}^{-1}
$$

where an incident flux spectrum of $(d \Phi / d E)$ is in units of $\left(\mathrm{cm}^{-2} \mathrm{~s}^{-1} \AA^{-1}\right)$, and the production of UCNs is up to the maximum storage Fermi energy of $160 \mathrm{neV}$. 32, 33] As shown in Fig. 1 the $8.9 \AA \mathrm{CN}$ flux in the SNS FnPB is about $5.9 \times 10^{7} \mathrm{CNs} \mathrm{cm}^{-2} \mathrm{~s}^{-1} \AA^{-1}$. The production rate per unit volume is deduced to be $R \approx 1.3 \mathrm{UCNs}$ $\mathrm{cm}^{-3} \mathrm{~s}^{-1}$. So the steady state UCN density $\rho_{U C N}$ can reach an average of about $141.4 \mathrm{UCNs} \mathrm{cm}^{-3}$ with $200 \mathrm{~s}$ of beam filling. i.e. a total number $N_{0} \approx 7.5 \times 10^{4}$ of UCNs can be filled in the storage cell with a beam-occupied volume of $530.1 \mathrm{~cm}^{3}$.

\section{A Possible Shortcut to Resolve Neutron Lifetime Enigma}

The capture and decay rates of UCNs in the storage volume are given by

$$
\begin{gathered}
\dot{N}_{p+t, U C N}(t)=-\frac{N_{0}}{\tau_{H e 3}} \exp \left(-\frac{t}{\tau_{t o t}}\right) \\
\dot{N}_{\beta, U C N}(t)=-\frac{N_{0}}{\tau_{\beta}} \exp \left(-\frac{t}{\tau_{t o t}}\right) \\
\kappa_{U C N}=\frac{\dot{N}_{p+t, U C N}\left(t_{1}\right)}{\dot{N}_{\beta, U C N}\left(t_{2}\right)}=\frac{\tau_{\beta}}{\tau_{H e 3}} \exp \left(-\frac{t_{1}-t_{2}}{\tau_{t o t}}\right)
\end{gathered}
$$

If the ${ }^{3} \mathrm{He}$ capture events can be distinguished from the decay events via the difference in the $1 / t$ decay rate of after-pulses, $\dot{N}_{p+t, U C N}(t)$ and $\dot{N}_{\beta, U C N}(t)$ can be directly acquired in the measurement. It is then convenient to obtain $\kappa_{U C N}$, the ratio of the capture-to-decay rate as in Eqn. (18). By counting both events in the identical time 
bins, $\kappa_{U C N}$ equals to $\tau_{\beta} / \tau_{H e 3}=\tau_{\beta} n_{H e 3} \sigma_{H e 3}^{t h} v_{H e 3}^{t h}$, which should statistically fluctuate around the true value. In this scenario, the neutron lifetime enigma can be resolved by simply comparing $\tau_{\beta} n_{H e 3}$ obtained in the current UCN storage measurement with that of Eqn. (3) in the beam decay volume. The real value of ${ }^{3} \mathrm{He}$ density $n_{H e 3}$ is not necessary.

\section{Accurate Determination of ${ }^{3} \mathrm{He}$ Density}

In order to determine the exact ${ }^{3} \mathrm{He}$ density, a comparison study on the UCN storage time has to be performed between $\tau_{\text {tot }}^{(0)}$ in the isotopically pure ${ }^{4} \mathrm{He}$ and $\tau_{\text {tot }}$ in the prepared liquid helium with a ${ }^{3} \mathrm{He}$ concentration of $X_{\mathrm{He} 3} \approx 2 \times 10^{-10}$. In both measurements, the total storage time is obtained by temporally binning scintillation event rates separately or together, and fitted as to Eqn. (16), 17), or 19, respectively, after normalization to the initial rate at time zero after the beam stops.

$$
\dot{N}_{t o t}(t)=-\left(\frac{N_{0}}{\tau_{H e 3}}+\frac{N_{0}}{\tau_{\beta}}\right) \exp \left(-\frac{t}{\tau_{t o t}}\right)
$$

For the isotopically pure ${ }^{4} \mathrm{He}, X_{H e 3}<2 \times 10^{-13}$, the ${ }^{3} \mathrm{He}$ capture lifetime is expected to be at least $2 \times 10^{5}$ seconds . The storage time $\tau_{\text {tot }}^{(0)}$ in pure ${ }^{4} \mathrm{He}$ is given by

$$
\tau_{\text {tot }}^{(0)-1}=\tau_{\beta}^{-1}+\tau_{\text {up }}^{-1}+\tau_{\text {loss }}^{-1}
$$

With the property of the UCN storage volume described in Subsection VII A the total storage time is expected to be about $551.7 \mathrm{~s}$, and the UCN density $\rho_{U C N}^{(0)}$ can reach about $219.2 \mathrm{UCNs} \mathrm{cm}^{-3}$, and with $200 \mathrm{~s}$ of beam filling, it achieves a fill of $N_{0} \approx 1.16 \times 10^{5} \mathrm{UCNs}$ in the storage volume.

Once the isotopically pure ${ }^{4} \mathrm{He}$ is confirmed, the desired ${ }^{3} \mathrm{He}$ concentration of about $X_{H e 3}=2 \times 10^{-10}$ can be prepared by mixing with natural helium of a known ${ }^{3} \mathrm{He}$ abundance as to a preset volume ratio. The ${ }^{3} \mathrm{He}$ atoms may be expelled from the volumes via heat flush to slightly reduce its concentration. 39 The exact ${ }^{3} \mathrm{He}$ concentration $X_{H e 3}$ is then determined via $\tau_{H e 3}^{-1}=\tau_{\text {tot }}^{-1}-\tau_{\text {tot }}^{(0)-1}=2.56 \times$ $10^{7} X_{H e 3} \mathrm{~s}^{-1}$. Adequate statistics is needed on both $\tau_{t o t}^{-1}$ and $\tau_{\text {tot }}^{(0)-1}$ to reach sub- $0.1 \%$ accuracy.

\section{E. Gamma Ray Backgrounds in the UCN Storage Measurement}

Similarly, gamma rays generated from the neutron captures on the surrounding materials are the major contributor to the background, but the UCN storage measurement is mostly sensitive to the delayed components rather than the prompt. In addition to the capture and scattering by the windows, the $8.9 \AA \mathrm{CN}$ beam has a scattering cross section of about 0.025 barns on liquid helium at $0.5 \mathrm{~K}$.
[40, 41] $4 \%$ of CNs will be inelastically scattered by the phonons in liquid helium. A very small portion is downconverted into UCNs and trapped in the storage volume, whereas majority of the scattered neutrons project at angles of around 84 degrees off the incident direction. About $12 \%$ of the scattered neutrons are captured by hydrogen, on top of those captured by the diamond window. The resultant prompt gamma rays are intensive but varnish right after the beam halts. Therefore, it doesn't affect the measurement of UCN storage time $\tau_{t o t}$. More troublesome are the delayed gamma rays from the $0.002 \%$ of scattered neutrons captured on the fluorine in the $50 \mu \mathrm{m}$ thick PTFE reflector. After $200 \mathrm{~s}$ of UCN filling with the $8.9 \AA \mathrm{CN}$ beam, the neutron-activated fluorine saturates. The resultant delayed gamma rays of $1.6 \mathrm{MeV}$ induce Compton scintillation at $5.0 \mathrm{~Hz}$ in liquid helium and $3.3 \mathrm{~Hz}$ in the fibers at time-zero when the beam turns off. Since the half-life of the activated fluorine is $11.16 \mathrm{~s}$, the rate of Compton scintillation in liquid helium drops below $1 \mathrm{~Hz}$ after $26 \mathrm{~s}$. At the mean time, the rate of both capture and decay events (or decay events solely) is $434.3 \mathrm{~Hz}$ (or $71.2 \mathrm{~Hz}$ ), assuming the storage time is $144.2 \mathrm{~s}$ and the initial UCN number is $N_{0} \approx 7.5 \times 10^{4}$. This type of delayed gamma ray background can be eliminated by replacing the PTFE reflector with a polymer reflector, such as Vikuiti VM2000 [42].

\section{CONCLUSION}

This proposed experiment has great potential to reach a sensitivity of $0.1 \%$ or sub- 1 second in neutron lifetime measurement. It offers an entirely different set of systematic uncertainties from the existing beam experiments. Most uniquely, it does not require any magnetic field. It may be set up to test the hypothesis of neutron-mirror neutron $n-n^{\prime}$ oscillations, where the intensity of magnetic field plays an important role. 43. Its apparent disadvantage is the flux of a CN beam at the wavelength $\lambda>16.5 \AA$ is much weaker than that of the most commonly in use 4-5 $\AA$ CN beams. It will take more beam time to gain adequate statistics. There might be a possibility to optimize the $\mathrm{CN}$ beam output for the long wavelength specifically needed in this experiment. Nevertheless, the low event rate allows a thorough characterization of the temporally spaced events with less interference. The measurement of ${ }^{3} \mathrm{He}$ density can be carried out parallel to that of the ratio of beam capture-to-decay event rates. The Atom Trap Analysis can be set up on the side of the apparatus with pops of samples exhaled by heating. In the method of counting scintillation of UCN captures and decays, the $8.9 \AA \mathrm{CN}$ beam may be separated from the main beam by a monochromator [17, 44] and sent to a connected twin volume of UCN storage beside the beam decay volume. Under proper arrangement, statistics on both the beam capture-to-decay ratio and the ${ }^{3} \mathrm{He}$ density can be gained simultaneously. Furthermore, while the intrinsic UCN storage time $\tau_{\text {tot }}^{(0)}$ is being measured in the storage volume 
filled with isotopically pure liquid ${ }^{4} \mathrm{He}$, the neutron beta decay spectrum can be simultaneously obtained in the decay volume at a good resolution and accuracy with this detector. It may provide a measurement on the Fierz interference term $b$ in the energy dependent neutron beta decay rate at a potentially high precision compared to the most recent results 45 .

\section{ACKNOWLEDGEMENT}

This paper greatly benefits from the publications, notes and data shared by the SNS nEDM collaboration. The author acknowledges Vince Cianciolo, Bradley W. Filippone, Roy J. Holt, Humphrey J. Maris, Jeffrey S. Nico, George M. Seidel, Christopher M. Swank, Fred E. Wietfeldt and Liyuan Zhang for help and discussions on many topics presented in this paper. This work is supported in part by the National Science Foundation under Grant No. 1812340.
[1] F. E. Wietfeldt and G. L. Greene, Colloquium: The neutron lifetime, Rev. Mod. Phys. 83, 1173 (2011)

[2] D. Dubbers and M. G. Schmidt, The neutron and its role in cosmology and particle physics, Rev. Mod. Phys. 83, $1111(2011)$

[3] F. E. Wietfeldt, Measurements of the Neutron Lifetime, Atoms 6, 70 (2018)

[4] J. Byrne, P. G. Dawber, C. G. Habeck, S. J. Smidt, J. A. Spain, and A. P. Williams, A revised value for the neutron lifetime measured using a Penning trap, Europhys. Lett., 33, 187 (1996)

[5] A. T. Yue, M. S. Dewey, D. M. Gilliam, G. L. Greene, A. B. Laptev, J. S. Nico, W. M. Snow, and F. E. Wietfeldt, Improved Determination of the Neutron Lifetime, Phys. Rev. Lett. 111, 222501 (2013)

[6] A. Serebrov, V. Varlamov, A. Kharitonov, A. Fomin, Y. Pokotilovski, P. Geltenbort, J. Butterworth, I. Krasnoschekova, M. Lasakov, R. Tal'daev, A.Vassiljev, and $\mathrm{O}$. Zherebtsov, Measurement of the neutron lifetime using a gravitational trap and a low-temperature Fomblin coating, Phys. Lett. B 605, 72 (2005)

[7] A. Pichlmaier, V. Varlamov, K. Schreckenbach, and P. Geltenbort, Neutron lifetime measurement with the UCN trap-in-trap MAMBO II, Phys. Lett. B 693, 221 (2010)

[8] A. Steyerl, J. Pendlebury, C. Kaufman, S. Malik, and A. Desai, Quasielastic scattering in the interaction of ultracold neutrons with a liquid wall and application in a reanalysis of the Mambo I neutron-lifetime experiment, Phys. Rev. C 85, 065503 (2012)

[9] S. Arzumanov, L. Bondarenko, S. Chernyavsky, P. Geltenbort, V. Morozov, V. V. Nesvizhevsky, Yu. Panin, and A. Strepetov, A measurement of the neutron lifetime using the method of storage of ultracold neutrons and detection of inelastically up-scattered neutrons, Phys. Lett. B 745, 79 (2015)

[10] A. P. Serebrov, E. A. Kolomensky, A. K. Fomin, I. A. Krasnoshchekova, A. V. Vassiljev, D. M. Prudnikov, I. V. Shoka, A. V. Chechkin, M. E. Chaikovskiy, V. E. Varlamov, S. N. Ivanov, A. N. Pirozhkov, P. Geltenbort, O. Zimmer, T. Jenke, M. Van der Grinten, and M. Tucker, Neutron lifetime measurements with a large gravitational trap for ultracold neutrons, Phys. Rev. C 97, 055503 (2018)

[11] R. W. Pattie Jr. et al., Measurement of the neutron lifetime using a magneto-gravitational trap and in situ detection, Science 360, 627 (2018)
[12] V. F. Ezhov,A. Z. Andreev, G. Ban, B. A. Bazarov, P. Geltenbort, A. G. Glushkov, V. A. Knyazkov, N. A. Kovrizhnykh, G. B. Krygin, O. Naviliat-Cuncic, and V. L. Ryabov, Measurement of the Neutron Lifetime with Ultracold Neutrons Stored in a Magneto-Gravitational Trap, J. Exp. Theor. Phys. Lett. 107, 707 (2018)

[13] N. Nagakura et al., Precise neutron lifetime experiment using pulsed neutron beams at J-PARC, arXiv:1702.03099 (2017)

[14] R. Kossakowski, P. Grivot, P. Liaud, K. Schreckenbach, G. Azuelos, Neutron lifetime measurement with a heliumfilled time projection chamber, Nucl. Phys. A503, 473 (1989)

[15] Z. Tang et al., Ultra-Cold Neutron measurement of Proton branching ratio in neutron Beta decay (UCNProBe), presentation H14.5, APS April Meeting, Denver, Colorado, 2019

[16] M. Cohen, and R. P. Feynman, Theory of Inelastic Scattering of Cold Neutrons from Liquid Helium, Phys. Rev. 107, 13 (1957)

[17] N. Fomin, G.L. Greene, R.R. Allen, V. Cianciolo, C. Crawford, T.M. Tito, P.R. Huffman, E.B. Iverson, R. Mahurin, and W.M. Snow, Fundamental neutron physics beamline at the spallation neutron source at ORNL, Nucl. Instrum. Meth. A 773, 45-51 (2015)

[18] P. C. Hendry and P. V. E. McClintock, Continuous flow apparatus for preparing isotopically pure ${ }^{4} \mathrm{He}$, Cryogen. 27, 131-138 (1987)

[19] J.S. Adams, A. Fleischmann, Y.H. Huang, Y.H. Kim, R.E. Lanou, H.J. Maris, and G.M. Seidel, Energy deposition by electrons in superfluid helium and design of a detector for solar neutrinos, Nucl. Instrum. Meth. A 444, 51 (2000)

[20] W. Guo and D. N. McKinsey, Concept for a dark matter detector using liquid helium-4, Phys. Rev. D 87, 115001 (2013)

[21] S. A. Hertel, A. Biekert, J. Lin, V. Velan, and D. N. McKinsey, Direct detection of sub-GeV dark matter using a superfluid helium-4 target, Phys. Rev. D 100, 092007 (2019)

[22] P.R. Huffman and D.N. McKinsey, Detecting scintillations in liquid helium, J. Instrum. 8, C09008 (2013)

[23] T. M. Ito, S. M. Clayton, J. Ramsey, M. Karcz, C.-Y. Liu, J. C. Long, T. G. Reddy, and G. M. Seidel, Effect of an electric field on superfluid helium scintillation produced by $\alpha$-particle sources, Phys. Rev. A 85, 042718 (2012)

[24] M. J. Berger, J. S. Coursey, M. A. Zucker, and J. Chang, 'Stopping-Power and Range Tables for Electrons, 
Protons, and Helium Ions, NIST Standard Reference database Number 124, 1998, National Institute of Standards and Technology, Gaithersburg, MD [http:// webbook.nist.gov].

[25] D. N. McKinsey, C. R. Brome, S. N. Dzhosyuk, R. Golub, K. Habicht, P. R. Huffman, E. Korobkina, S. K. Lamoreaux, C. E. H. Mattoni, A. K. Thompson, L. Yang, and J. M. Doyle, Time dependence of liquid-helium fluorescence, Phys. Rev. A 67, 062716 (2003);

D. N. McKinsey, Detection of Magnetically Trapped Neutrons: Liquid Helium as a Scintillator, Ph.D. thesis, Harvard University (2002)

[26] D. N. McKinsey, C. R. Brome, J. S. Butterworth, S. N. Dzhosyuk, R. Golub, K. Habicht, P. R. Huffman, C. E. H. Mattoni, L. Yang, and J.M. Doyle, Detecting ionizing radiation in liquid helium using wavelength shifting light collection, Nucl. Instrum. Meth. A 516, 475 (2004)

[27] W. Guo, M. Dufault, S. B. Cahn, J. A. Nikkel, Y. Shin, and D. N. McKinsey, Scintillation and charge yield from the tracks of energetic electrons in superfluid helium-4, J. Instrum. 7, P01002 (2012).

[28] D. N. McKinsey, C. R. Brome , J. S. Butterworth, R. Golub , K.Habicht, P. R. Huffman, S. K. Lamoreaux, C. E. H. Mattoni, and J. M. Doyle, Fluorescence efficiencies of thin scintillating films in the extreme ultraviolet spectral region, Nucl. Instrum. Meth. B 132, 351 (1997)

[29] Plastic Scintillating Fibers by Kuraray Co., Ltd., http: //kuraraypsf.jp

[30] Red-enhanced silicon photomultipliers, MICROB-10035, by ON Semiconductor, http://www.onsemi.com

[31] J. S. Adams, Energy Deposition by Electrons in Superfluid Helium, Ph.D. thesis, Brown University (2001)

[32] R. Golub and J. M. Pendlebury, The interaction of ultracold neutrons (UCN) with liquid helium and a superthermal UCN source, Phys. Lett. A 62 , 337 (1977)

[33] C. A. Baker et al., Experimental measurement of ultracold neutron production in superfluid He-4, Phys. Lett. A 308, $67(2003)$

[34] Polycrystalline CVD diamond windows, http://www iiviinfrared.com/

[35] B. Plaster et al., Measurement of the neutron $\beta$ asymmetry parameter AO with ultracold neutrons, Phys.
Rev. C 86, $055501(2012)$

[36] W. Jiang, W. Williams, K. Bailey, A. M. Davis, S.-M. Hu, Z.-T. Lu, T. P. O'Connor, R. Purtschert, N. C. Sturchio, Y. R. Sun, and P. Mueller, ${ }^{39}$ Ar Detection at the $10^{-16}$ Isotopic Abundance Level with Atom Trap Trace Analysis, Phys. Rev. Lett. 106, 103001 (2011)

[37] K. K. H. Leung et al., to be published; Z. Tang et al., to be published

[38] M. W. Ahmed et al., A new cryogenic apparatus to search for the neutron electric dipole moment, J. Instrum. 14, P11017 (2019)

[39] G. Baym, D. H. Beck, and C. J. Pethick, Transport in ultradilute solutions of ${ }^{3} \mathrm{He}$ in superfluid ${ }^{4} \mathrm{He}$, Phys. Rev. B 92, 024504 (2015)

[40] H. S. Sommers Jr., J.G. Dash, and L. Goldstein, Transmission of Slow Neutrons by Liquid Helium, Phys. Rev. 97, 855 (1955)

[41] Y. Abe and N. Morishima, Ultracold and cold neutron cross-sections of liquid helium at low temperatures down to $0.1 \mathrm{~K}$, Nucl. Instrum. Meth. A 459, 256 (2001)

[42] Vikuiti VM2000, dielectric mirror film, http://www apioptics.com/pdf/ESR.pdf

[43] Z. Berezhiani, Neutron lifetime puzzle and neutron-mirror neutron oscillation, Eur. Phys. J. C 79, 484 (2019); W. P. Tan, Neutron oscillations for solving neutron lifetime and dark matter puzzles, Phys. Lett. B 797, 134921 (2019)

[44] C. E. H. Mattoni, C. P. Adams, K. J. Alvine, J. M. Doyle, S. N. Dzhosyuk, R. Golub, E. Korobkina, D. N. McKinsey, A. K. Thompson, L. Yang, H. Zabel, and P. R. Huffman, A long wavelength neutron monochromator for superthermal production of ultracold neutrons, Phys. B Conden. Matt. 344, 343 (2004)

[45] H. Saul, C. Roick, H. Abele, H. Mest, M. Klopf, A. Petukhov, T. Soldner, X. Wang, D. Werder, B. Markisch, Limit on the Fierz Interference Term $b$ from a Measurement of the Beta Asymmetry in Neutron Decay, arXiv:1911.01766 (2019);

$\mathrm{X}$. Sun et al., Improved limits on Fierz Interference using asymmetry measurements from the UCNA experiment, arXiv:1911.05829 (2019) 